\title{
Efeito da elastocompressão no edema de membros inferiores em profissionais da saúde após jornada vespertina de trabalho em centro cirúrgico
}

\author{
Effect of elastocompression in edema of lower limbs in health professionals after \\ an afternoon work day in a surgical center
}

\author{
Melissa Andreia de Moraes Silva ${ }^{1}$, Brenda Mayra Fernandes de Carvalho ${ }^{2}$, \\ Seleno Glauber de Jesus Silva ${ }^{3}$, Cleusa Ema Quilici Belczak ${ }^{4}$, Rodolfo Souza Cardoso 5
}

de Moraes Silva MA, Carvalho BMF, Jesus-Silva SG, Belczac CEQ, Cardoso RC. Efeito da elastocompressão no edema de membros inferiores em profissionais da saúde após jornada vespertina de trabalho em centro cirúrgico / Effect of elastocompression on edema of lower limbs in health professionals after an afternoon work day in a surgical center. Rev Med (São Paulo). 2018 set.-out.;97(5):469-75.

RESUMO: Introdução: $\mathrm{O}$ edema de membros inferiores é um sinal precoce de insuficiência venosa crônica, já estando bem estabelecida a relação entre sua formação e atividades laborais extenuantes. Dessa forma, medidas que previnam e minimizem a sua formação devem ser adotadas, objetivando promoção à saúde do trabalhador e prevenção da IVC. O uso de meias elásticas durante o trabalho é uma medida simples e eficaz. Objetivos: Demonstrar os efeitos do uso de meias elásticas na redução do edema em membros inferiores formado durante atividade laboral em profissionais de saúde. Métodos: Foram analisadas 7 funcionárias técnicas de enfermagem do centro cirúrgico de um hospital quaternário, com idade entre 21 e 41 anos, com jornada de trabalho de 6 horas no período vespertino. As participantes foram questionadas sobre sintomas, além de terem tido mensurados os perímetros das regiões metatarsal, maleolar e de panturrilha e o volume dos membros inferiores, antes e após o trabalho, em duas etapas, uma sem o uso de meias de elastocompressão e outra com o uso das mesmas, através de volumetria por deslocamento de água. Resultados: Pode-se observar aumento no perímetro e volume dos membros inferiores, após trabalho sem uso de meias elásticas e redução dos mesmo com o uso da elastocompressão. Conclusão: A utilização de meias de elastocompressão, durante jornada de trabalho vespertino, promoveu redução no edema de membros inferiores além de melhora nos sintomas como dores, cansaço e sensação de peso nas pernas.

Descritores: Meias de compressão; Edema; Jornada de trabalho; Doenças vasculares.
ABSTRACT: Introduction: Lower limb edema is an early sign of chronic venous insufficiency, and the relationship between its formation and strenuous work activities is well established. Thus, measures that prevent and minimize their formation should be adopted, aiming at promoting worker health and prevention of CVI. The use of elastic stockings during work is a simple and effective measure. Objectives: To demonstrate the effects of the use of stocking compression in the reduction of edema in lower limbs formed during work activity in health professionals. Methods: Seven nursing technicians from the surgical center of a quaternary hospital, aged between 21 and 41 years, with a workday of 6 hours in the evening period, were analyzed. Participants were questioned about symptoms, in addition to having measured the perimeters of the metatarsal, malleolar and calf regions and the volume of the lower limbs, before and after work, in two steps, one without the use of elasto compression and another with the use of the same, through volumetric displacement of water. Results: An increase in the perimeter and volume of the lower limbs can be observed after work without the use of elastic stockings and reduction of the same with the use of elasto compression. Conclusion: The use of stocking compression during the afternoon workday promoted a reduction in lower limb edema and an improvement in symptoms such as pain, tiredness and weight sensation in the legs.

Keywords: Stockings, compression; Edema; Work hours, Vascular diseases.

Instituição: Faculdade de Medicina de Itajubá (FMIt) - Itajubá/MG. Trabalho apresentado no IV Congresso Brasileiro das Ligas de Cirurgia, SP, 27 jan. 2018.

1. Cirurgiã Vascular. Especialista em Cirurgia Vascular e Ultrassonografia Vascular. Mestre em Ciências pela Universidade Federal de São Paulo. Professora Assistente da Disciplina de Clínica Cirúrgica da Faculdade de Medicina de Itajubá (FMIt). https://orcid.org/0000-0001-8773-3095. E-mail: meldemoraes@gmail.com.

2. Acadêmica do $6^{\circ}$ ano do curso de medicina da Faculdade de Medicina de Itajubá/MG (FMIt). https://orcid.org/0000-0003-1106-5443. E-mail: brendafernandis@gmail.com.

3. Cirurgião Vascular. Especialista em Cirurgia Vascular e Angiorradiologia. Preceptor do Serviço de Residência Médica em Cirurgia Vascular do Hospital de Clínicas de Itajubá-MG. https://orcid.org/0000-0002-2205-1090. E-mail: selenoglauber@gmail.com.

4. Doutora em Cirurgia Geral pela Faculdade de Ciências Médicas da Santa Casa de São Paulo (FCMSCSP) e Diretora do Centro Vascular João Belczak, Maringá - PR. ORCID: https://orcid.org/0000-0003-2143-701X. Email: belczak@wnet.com.br.

5. Cirurgião Vascular. Especialista em Cirurgia Vascular e Angiorradiologia. Preceptor(a) do Serviço de Residência Médica em Cirurgia Vascular do Hospital de Clínicas de Itajubá-MG. ORCID: Cardoso RS - https://orcid.org/0000-0003-0212-240X; Belzack CQ - https://orcid.org/0000-0003-2143701X. E-mail: rodolfosc@globo.com.

Endereço para correspondência: Melissa A. de Moraes Silva. FMIt. Av. Renó Júnior, 368. Bairro São Vicente. Itajubá, MG. CEP: 37502-138. E-mail: meldemoraes@gmail.com. 


\section{INTRODUÇÃO}

A insuficiência venosa crônica (IVC) é Aconsiderada importante problema de saúde pública acometendo pessoas de diferentes faixas etárias, gerando sérios problemas socioeconômicos, como, por exemplo, a inaptidão para o trabalho ${ }^{1}$. Apresenta prevalência alta na população e pode ser definida como uma disfunção no sistema venoso decorrente da hipertensão nestes vasos, que ocorre devido a incompetência valvular e/ou obstrução do fluxo sanguíneo nas veias². Estima-se que até $60 \%$ das mulheres e até $55 \%$ dos homens sejam acometidos por tal doença ${ }^{3}$.

A IVC pode se manifestar por meio de diversos sinais e sintomas que variam de simples dilatações varicosas nos membros inferiores, como sensação de peso, dermatite ocre, lipodermatoesclerose, edema crônico e úlceras de estase $^{2}$. Diversas condições como postura, jornadas de trabalho extenuantes e reduzidas pausas para repouso são consideradas fatores agravantes para tal doença ${ }^{4}$.

A correlação entre posturas laborais adotadas por longos períodos durante a jornada diária de trabalho e o aumento de volume dos membros inferiores (MMII) já está bem estabelecida, e isto ocorre inclusive em indivíduos sem sinais e sintomas de IVC5.

Os sujeitos que trabalham em uma profissão que requer uma permanência prolongada são considerados como alto risco para desenvolvimento de sintomas em membros inferiores, sendo sugerido que os mesmos estejam sujeitos a um risco elevado de desenvolvimento de $\mathrm{IVC}^{6}$.

O uso de meias elásticas de compressão (MEC) tem sido neste contexto, de grande utilidade. Há evidências científicas que demonstram os benefícios na utilização das mesmas.

\section{OBJETIVOS}

Demonstrar os efeitos do uso de meias elásticas em membros inferiores formado durante atividade laboral vespertina em profissionais de saúde.

\section{MÉTODOS}

O presente estudo obteve aprovação do comitê de ética em pesquisa, sob parecer 1.785.675. Foram incluídas no estudo 7 funcionárias, todas técnicas de enfermagem, com idade entre 21 e 41 anos e jornada diária de trabalho de 6 horas no período vespertino, cujas profissões e atividades realizadas impõem postura predominantemente ortostática. As funcionárias aceitaram participar do experimento, com assinatura de Termo de Consentimento Livre e Esclarecido.

As avaliações ocorreram em dois dias distintos: um quando trabalharam sem MEC e outro com o uso de MEC (Comfortline ${ }^{\circledR}$, Venosan, Abreu e Lima, Brasil) (Figura 1). Para se avaliar as alterações de volume dos membros inferiores realizaram-se medidas dos membros inferiores por meio de perimetria e volumetria.
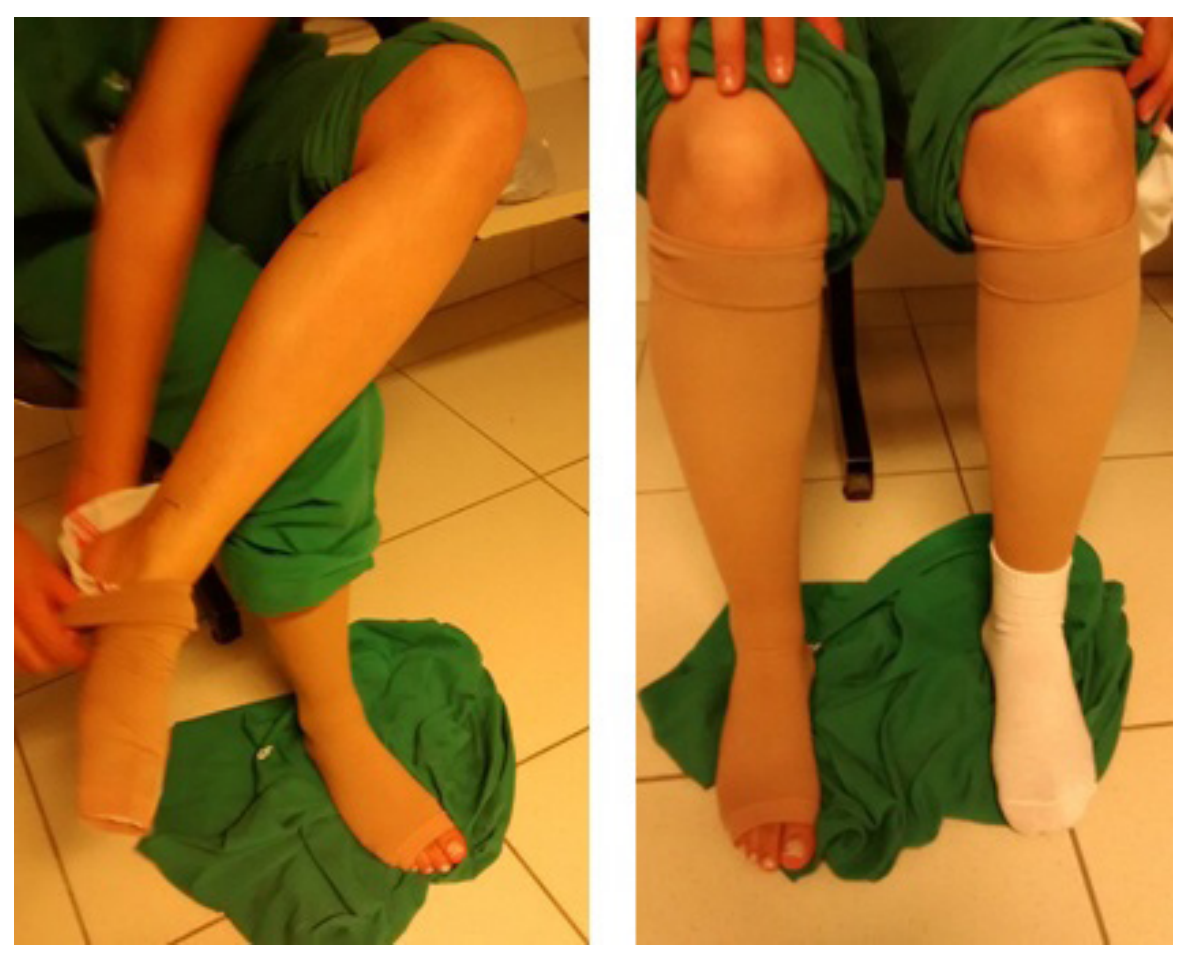

Figura 1. Colocação das meias elásticas de média compressão antes do início das atividades laborais 
A perimetria foi realizada com auxílio de fita métrica, com a coleta do comprimento das circunferências das pernas direita e esquerda de cada uma das funcionárias, nas regiões metatarsal, maleolar e de panturrilha. O volume de cada uma das pernas foi aferido por meio de técnica de volumetria por deslocamento de água.
A técnica consiste na imersão de cada uma das pernas individualmente, em cuba de acrílico contendo água e uma válvula para saída da mesma (Figura 2). O volume de água deslocado, após imersão de cada perna, é devidamente capturado e posteriormente medido, correspondendo ao volume de cada um dos membros.
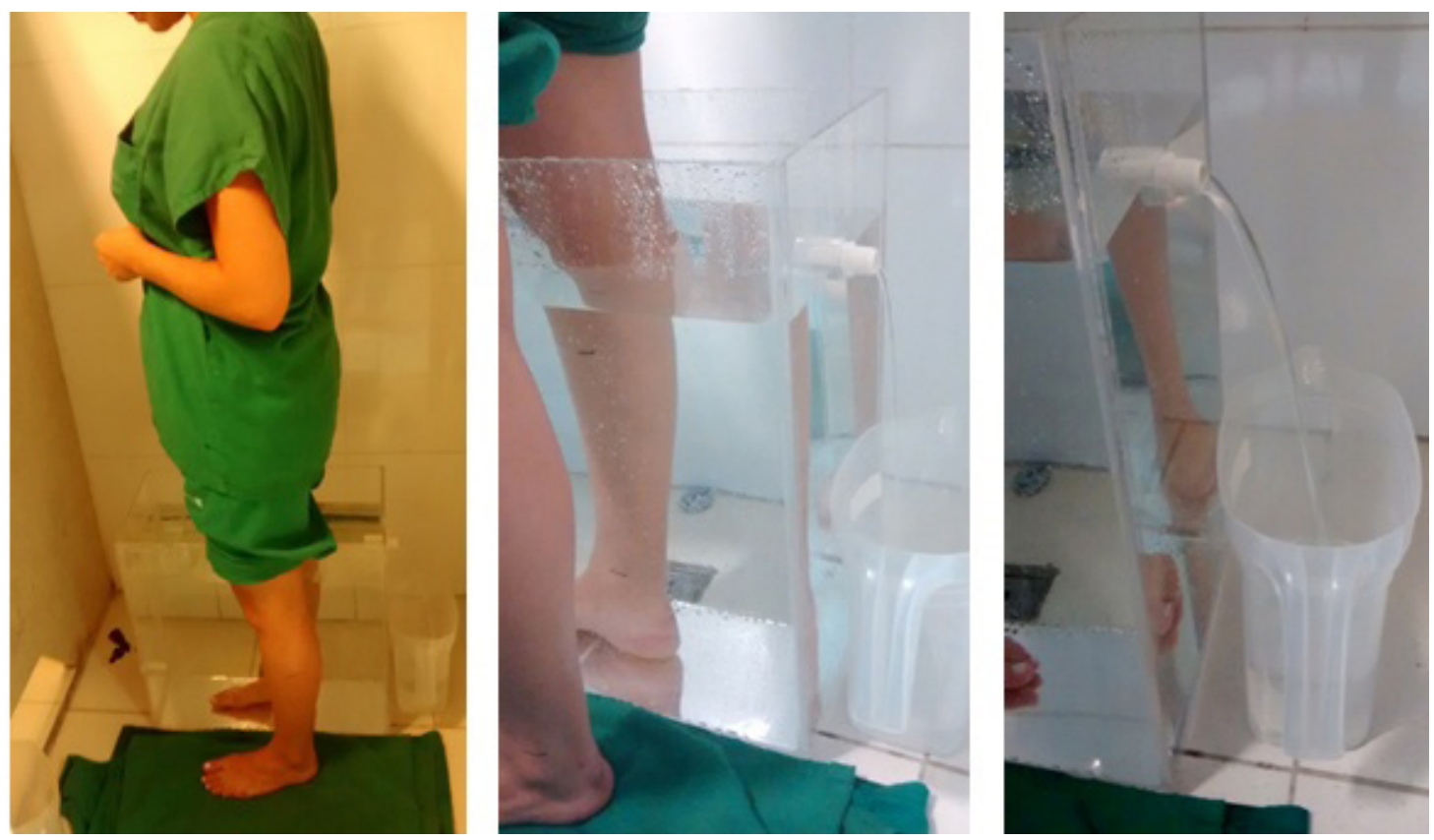

Figura 2. Realização da técnica de volumetria por deslocamento de água

Todas as medidas foram realizadas antes e após jornada de trabalho.

As participantes foram orientadas a realizar as atividades de rotina nos dias do experimento e também foram questionadas antes e após jornada de trabalho, sobre possíveis sintomas nos membros inferiores, como dor, edema, sensação de peso e sintomas não usuais.

Para avaliação da média para idade, peso e IMC dos participantes, foram utilizados valores de média aritmética considerando desvio padrão. Para a comparação dos resultados entre perimetria e volumetria e lactato sanguíneo utilizou-se o Teste de Wilcoxon. Todos os testes consideraram $\mathrm{p}$ significativo $<0,05$.

\section{RESULTADOS}

Foram selecionadas inicialmente 8 voluntárias, porém uma delas não completou o estudo pois foi transferida de setor antes da coleta dos dados com o uso das MEC. Todas são do sexo feminino, apresentando a média de idade de 29,8 anos ( $\pm 5,5$ anos), média de peso de 73,21 $\mathrm{kg}( \pm 9,72 \mathrm{~kg})$, média de IMC de $26,76 \mathrm{~kg} / \mathrm{m}^{2}( \pm 3,05 \mathrm{~kg} /$ $\mathrm{m}^{2}$ ). Apenas duas realizam atividade física regularmente, sem relato de dupla jornada de trabalho.

Os resultados referentes aos sintomas nos membros inferiores, obtidos mediante entrevista com as voluntárias do estudo, são apresentados na Tabela 1. Observou-se maior prevalência de sintomas incômodos nos membros inferiores após jornada de trabalho sem o uso de meias de elastocompressão e redução dos mesmos após jornada de trabalho com uso das meias.

Tabela 1. Prevalência dos sintomas apresentados pelas voluntárias do estudo, antes e após a jornada de trabalho sem e com o uso de meias de elastocompressão

\begin{tabular}{lcc}
\hline & $\begin{array}{c}\text { Antes da } \\
\text { jornada de } \\
\text { trabalho }\end{array}$ & $\begin{array}{c}\text { Após jornada } \\
\text { de trabalho }\end{array}$ \\
\hline Sem meia & $42,86 \%$ & $85,71 \%$ \\
Dor & $28,57 \%$ & $71,43 \%$ \\
Edema & $42,86 \%$ & $71,43 \%$ \\
Sensação de peso & $0,00 \%$ & $28,57 \%$ \\
Sintomas não usuais & & \\
Com meia & $42,86 \%$ & $0,00 \%$ \\
Dor & $42,86 \%$ & $28,57 \%$ \\
Edema & $42,86 \%$ & $0,00 \%$ \\
Sensação de peso & $28,57 \%$ & $0,00 \%$ \\
Sintomas não usuais & & \\
\hline
\end{tabular}


A Tabela 2 apresenta os valores médios dos perímetros das regiões metatarsal, de tornozelo e de panturrilha, antes e após jornada de trabalho, nas etapas com e sem o uso de MEC.

Tabela 2. Perímetros médios das regiões metatarsal, de tornozelo e de panturrilha em MMII, antes e após jornada de trabalho, com e sem o uso de meias de elastocompressão

\begin{tabular}{|c|c|c|c|c|}
\hline & Antes (cm) & Depois (cm) & Diferença & $\mathbf{p}$ \\
\hline \multicolumn{5}{|l|}{ Sem Meia } \\
\hline \multicolumn{5}{|l|}{ Direita } \\
\hline Metatarso & 20,8 & 21,0 & 0,2 & 0,250 \\
\hline Tornozelo & 20,7 & 21,1 & 0,4 & 0,063 \\
\hline Panturrilha & 35,3 & 35,8 & 0,5 & 0,031 \\
\hline \multicolumn{5}{|l|}{ Esquerda } \\
\hline Metatarso & 21,0 & 21,4 & 0,4 & 0,250 \\
\hline Tornozelo & 21,0 & 21,6 & 0,6 & 0,031 \\
\hline Panturrilha & 36,1 & 36,6 & 0,5 & 0,031 \\
\hline \multicolumn{5}{|l|}{ Com Meia } \\
\hline \multicolumn{5}{|l|}{ Direita } \\
\hline Metatarso & 21,3 & 20,7 & $-0,6$ & 0,063 \\
\hline Tornozelo & 21,1 & 20,5 & $-0,6$ & $\mathbf{0 , 0 3 1}$ \\
\hline Panturrilha & 35,5 & 34,9 & $-0,6$ & 0,063 \\
\hline \multicolumn{5}{|l|}{ Esquerda } \\
\hline Metatarso & 21,6 & 21,2 & $-0,4$ & 0,063 \\
\hline Tornozelo & 20,9 & 20,9 & 0 & 0,788 \\
\hline Panturrilha & 36,1 & 35,3 & $-0,8$ & 0,031 \\
\hline
\end{tabular}

Os dados apresentados na Tabela 2 são representados por meio de gráficos de boxplot (Figuras 3 e 4). A Figura 3 evidencia que ocorreu aumento significativo nas médias das circunferências da panturrilha direita $(\mathrm{p}=0,031)$ (Figura 3a) e do tornozelo esquerdo $(\mathrm{p}=0,031)$ e panturrilha esquerda $(\mathrm{p}=0,031)$ (Figura $3 \mathrm{~b})$, após jornada de trabalho sem o uso de meias elásticas. (a)

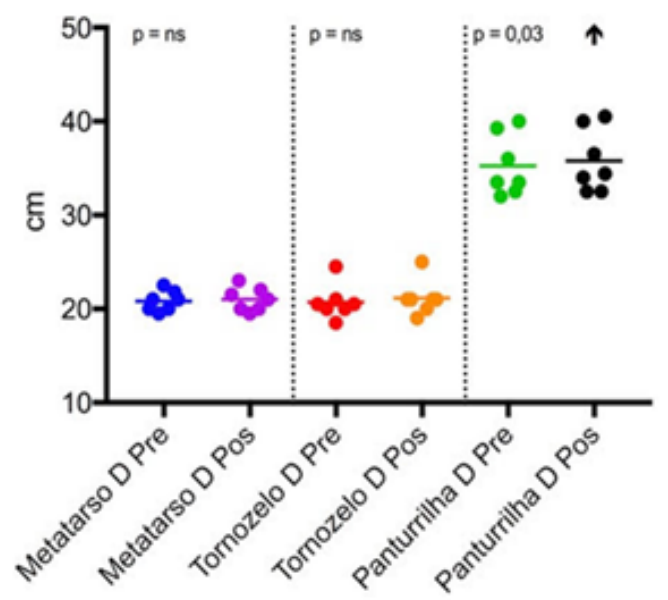

(b)

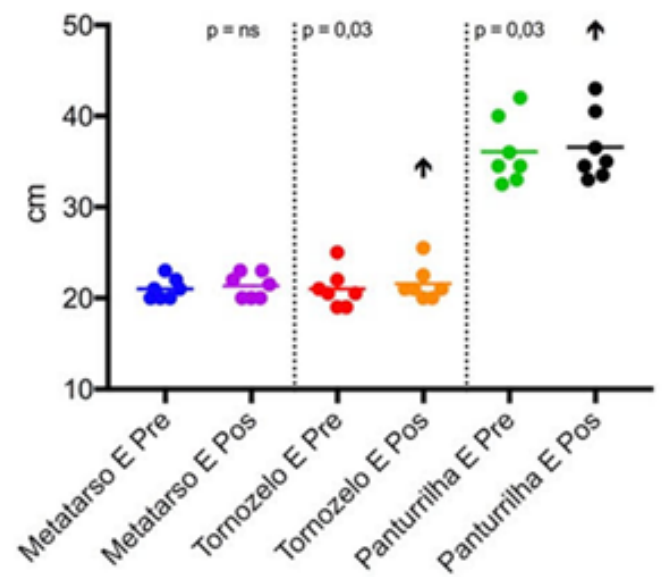

Figura 3. Gráfico de boxplot referente aos perímetros médios dos membros inferiores direito (a) e esquerdo (b), nas regiões metatarsal, de tornozelo e panturrilha, antes a após jornada de trabalho, sem a utilização de MEC 
Resultado oposto foi obtido com o uso das meias de elastocompressão, em que se observa por meio da Figura 4 uma redução significativa nos perímetros médios do tornozelo direito $(\mathrm{p}=0,031)$ (Figura $4 \mathrm{a})$, e panturrilha esquerda $(p=0,031)$ (Figura $4 b)$, após jornada de trabalho. (a)

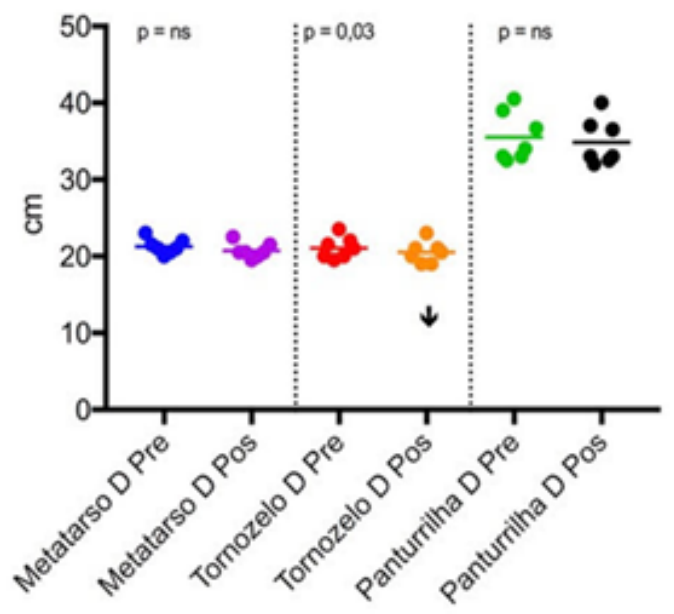

(b)

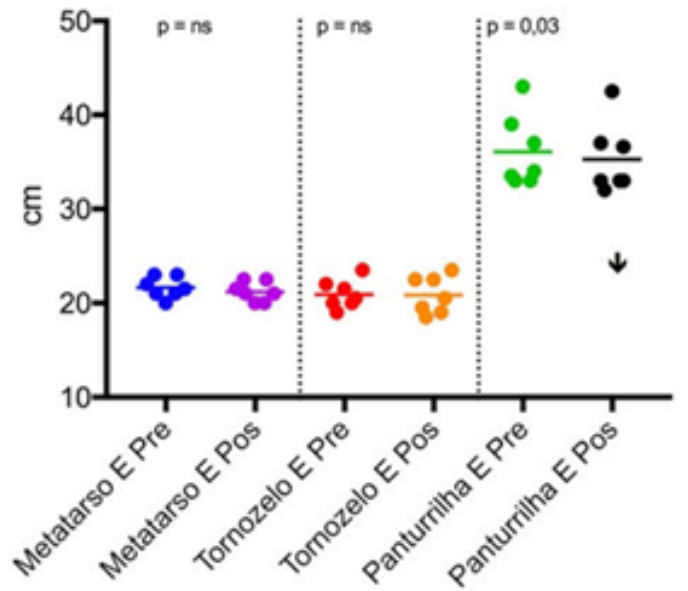

Figura 4. Gráfico de boxplot referente aos perímetros médios dos membros inferiores direito (a) e esquerdo (b), nas regiões metatarsal, de tornozelo e panturrilha, antes e após jornada de trabalho, com a utilização de meias de elastocompressão

A Tabela 3 apresenta os valores médios dos volumes dos membros inferiores direito e esquerdo obtidos antes e após jornada de trabalho, nas etapas com e sem o uso de meias de elastocompressão. Os dados da tabela estão resumidos na Figura 5, e conforme pode-se observar abaixo, constatou-se um aumento significativo dos volumes médios dos membros inferiores direito $(\mathrm{p}=0,016)$ e esquerdo $(\mathrm{p}=0,047)$ (Figura 5a), após jornada de trabalho sem o uso de meias elásticas e uma redução significativa dos mesmos com o uso de tais meias (Figura 5b).
Tabela 3. Volumes dos membros inferiores direito e esquerdo, antes e após jornada de trabalho, com e sem o uso de meias de elastocompressão

\begin{tabular}{lcccc}
\hline & Antes $(\mathbf{m L})$ & Depois $(\mathbf{m L})$ & Diferença & $\mathbf{p}$ \\
\hline Sem Meia & & & & \\
Direita & 3165,14 & 3213,29 & 48,14 & 0,016 \\
Esquerda & 3301 & 3353,71 & 52,71 & 0,047 \\
Com Meia & & & & \\
Direita & 3167 & 3131,71 & $-35,28$ & 0,047 \\
Esquerda & 3260 & 3221,57 & $-38,43$ & 0,032 \\
\hline
\end{tabular}

(a)

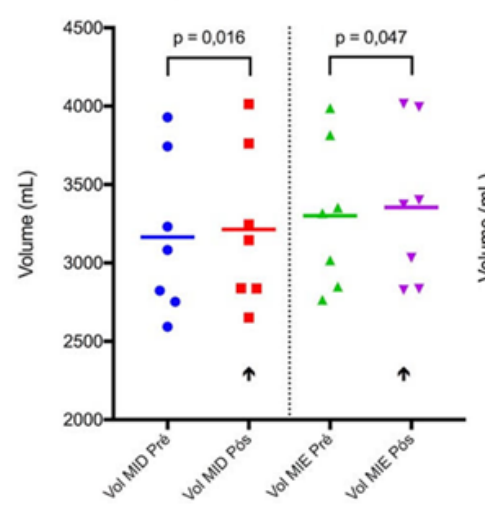

(b)

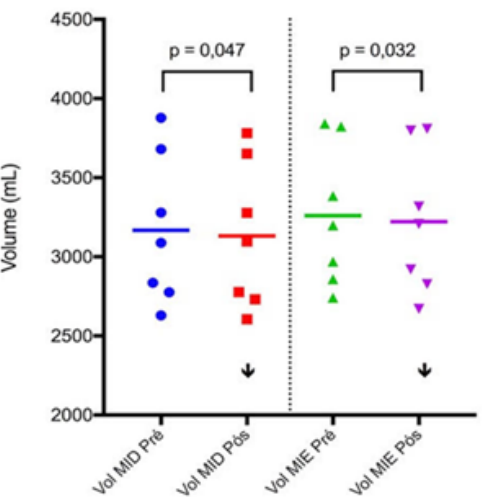

Figura 5. Gráfico de boxplot referente aos volumes médios dos membros inferiores direito e esquerdo antes e após jornada de trabalho, sem a utilização de meias de elastocompressão (a) e com o uso de tais meias (b) 


\section{DISCUSSÃO}

$\mathrm{O}$ atual estudo demonstrou que o a jornada de trabalho vespertino promove edema e relato de sintomas em membros inferiores, e que o uso de MEC, de modo inverso, acarreta diminuição de diâmetros e volume de membros inferiores, além de promover melhora de sintomas como dor e sensação de peso.

O edema ocupacional geralmente é assintomático, mas pode ser associado a sentimentos de cansaço, peso das pernas e dor ${ }^{7}$, que podem evoluir de diversas formas, causando prejuízo na qualidade de vida dos indivíduos.

Houve diminuição dos relatos de dor, edema e sensação de peso após a jornada de trabalho no momento que as voluntarias utilizaram a MEC. Esse achado está de acordo com os resultados de um estudo controlado randomizado que demonstrou melhora dos sintomas subjetivos após o uso de meias de compressão médica de 10 a $15 \mathrm{mmHg}$, quando comparado com placebo ${ }^{8}$.

Sabe-se que longos períodos em ortostatismo são fatores de risco para o desenvolvimento da DVC, e diversas atividades laborais exigem tal postura. Nesse contexto, diversos estudos têm investigado o edema ocupacional de membros inferiores e a associação deste com sinais e sintomas da IVC ${ }^{9}$. Outros estudos avaliam a formação de edema relacionado à posturas e profissões diversas, no entanto não se pesquisou a influência do uso de meias de elastocompressão na redução do edema formado. Em todos os trabalhos os autores puderam estabelecer a relação pretendida, evidenciando-se aumento no edema de membros inferiores relacionado ao ortostatismo e às jornadas de trabalho extenuantes ${ }^{1,5,10}$.

No trabalho realizado por Blazec et al. ${ }^{6}$ avaliaram 108 cabeleireiros, com atividades laborais predominantemente em posição ortostática, os quais foram divididos igualmente em grupos A e B. O grupo A realizou suas atividades durante três semanas fazendo uso de meias de elastocompressão, enquanto no mesmo período o grupo $\mathrm{B}$ não as utilizou. Observou-se redução dos sintomas após o uso das MEC nos dois grupos, $13,2 \%$ no grupo A e $11,6 \%$ no grupo B. Também se observou redução no volume médio dos membros inferiores (redução de cerca de $19 \mathrm{~mL}$ ), corroborando os resultados encontrados no presente trabalho.

Existem diversas limitações no estudo produzido, como a pequena amostra utilizada, o curto período para sua realização a interferência das atividades realizadas no período matutino, anterior ao trabalho.

Entretanto, poucos estudos analisam o uso ou não de MEC em pessoas saudáveis ${ }^{6,7,11}$, porem todos mostraram algum tipo de benefício com o uso da elastocompressão. $\mathrm{O}$ que não foi identificado na literatura foi o mesmo tipo de leitura que realizamos, usando a meia apenas no período vespertino.

\section{CONCLUSÃO}

A utilização de meias de elastocompressão, durante jornada de trabalho vespertino, promoveu redução no edema de membros inferiores além de melhora nos sintomas como dores, cansaço e sensação de peso nas pernas.

Agradecimentos: À FAPEMIG, pelo apoio no desenvolvimento deste trabalho, por meio da concessão de bolsa de Iniciação Científica e à Venosan, que gentilmente cedeu as meias elásticas utilizadas na pesquisa.

Indicação sobre a contribuição de cada autor: MAMS: Concepção e desenho do estudo. BMFC: Análise e Interpretação dos dados. SGJS, MAMS, BMFC: Coleta de dados. BMFC, MAMS: Redação do manuscrito. MAMS, SGJS, RSC: Revisão crítica do texto. MAMS, SGJS, BMFC, AEK, RSC: Aprovação final do manuscrito. SGJS, MAMS: Análise estatística. MAMS: Responsabilidade geral pelo estudo.

\section{REFERÊNCIAS}

1. Berenguer FA, Silva DAL, de Carvalho CC. Influência da posição ortostática na ocorrência de sintomas e sinais clínicos de venopatias de membros inferiores em trabalhadores de uma gráfica na cidade do Recife-PE. Rev Bras Saúde Ocup. 2011;36(123):153-61. doi: 10.1590/s030376572011000100016.

2. Castro e Silva M, Cabral ALS, Barros Jr N, Castro AA, Santos MERC. Diagnóstico e tratamento da doença venosa crônica. J Vasc Bras. 2005;4(3):S185-94. Disponível em: http://jvascbras. com.br/pdf/Arquivo_1.pdf.

3. Melo BV, Tojal PGMD, de Jesus Leal F, Couto RC. Quality of life in chronic venous patients who do or do not wear compressive stockings. J Vasc Bras. 2015;14(1):62-7. doi: 10.1590/1677-5449.20140070.

4. Belczak CEQ, Godoy JMP, Ramos RN, Oliveira MA,
Belczak SQ, Caffaro RA. Influência do turno laboral na formação de edema dos membros inferiores em indivíduos normais. J Vasc Bras. 2008;7(3):225-30. doi: 10.1590/S167754492008000300007.

5. Belczak CEQ, Godoy JMP, Seidel AC, Ramos RN, Belczak SQ, Caffaro RA. Influence of prevalent occupational position during working day on occupational lower limb edema. J Vasc Bras. 2015;14(2):153-60. doi: 10.1590/1677-5449.0079.

6. Blazek C, Amsler F, Blaettler W, Keo HH, Baumgartner I, Willenberg T. Compression hosiery for occupational leg symptoms and leg volume: a randomized crossover trial in a cohort of hairdressers. Phlebology. 2013;28(5):239-47. doi: 10.1258/phleb.2011.011108.

7. Wou J, Williams KJ, Davies AH. Compression Stockings versus Neuromuscular Electrical Stimulation Devices in the Management of Occupational Leg Swelling. Int J Angiol. 2016 Jun;25(2):104-9. doi: 10.1055/s-0035-1558646. 
8. Benigni J-P, Sadoun S, Allaert FA, Vin F. Efficacy of Class 1 elastic compression stockings in the early stages of chronic venous disease. A comparative study. Int Angiol. 2003;22(4):383-92. Available from: https://www. minervamedica.it/en/journals/international-angiology/article. php?cod=R34Y2003N04A0383\&acquista $=1$.

9. Belczak CEQ, de Godoy JMP, Ramos RN, de Oliveira MA, Belczak SQ, Caffaro RA. Rate of occupational leg swelling is greater in the morning than in the afternoon. Phlebology.
2009;24(1):21-5. doi: 10.1258/phleb.2008.008042

10. Brito A, Rodrigues E, Vianna DL. Investigation of postural edema in the lower extremities of traffic control workers. J Vasc Bras. 2013;12(4):289-95. doi: 10.1590/jvb.2013.055.

11. Mosti G, Partsch H. Occupational Leg oedema is more reduced by antigraduated than by graduated stockings. Europ J Vasc Endovasc Surg. 2013;45(5):523-7. doi: 10.1016/j. ejvs.2013.01.032.

Submetido em: 25.02.2018

Aceito em: 05.10.2018 